\title{
EFFICIENT EVALUATION OF PERFORMANCE-BASED EARTHQUAKE ENGINEERING EQUATIONS
}

\author{
Brendon A Bradley ${ }^{1 *}$, Dominic S Lee ${ }^{2}$, Robert Broughton ${ }^{2}$, Christopher Price ${ }^{2}$. \\ ${ }^{1}$ Department of Civil Engineering, University of Canterbury, Private Bag 4800, Christchurch 8020, New Zealand \\ ${ }^{2}$ Department of Mathematics and Statistics, University of Canterbury, Private Bag 4800, Christchurch 8020, New \\ Zealand \\ *Corrosponding author: $\mathrm{Ph}+64-3-3667001$ ext 7333; Fax: +64-3-364 2758; \\ Email: bab54@student.canterbury.ac.nz
}

\begin{abstract}
In this paper attention is given to the efficient numerical evaluation of the Pacific Earthquake Engineering Research (PEER) performance-based earthquake engineering framework equations. In particular, potential problems in determining an adequate yet efficient region of integration are discussed. An algorithm called "Magnitude-oriented Adaptive Quadrature" (MAQ) is developed, which is an integration algorithm with both locally and globally adaptive capabilities. MAQ allows efficient integration over the entire integration domain and requires only an error tolerance and maximum number of function evaluations to be specified. The advantages of utilizing the MAQ algorithm over other conventional integration methods such as Romberg integration and conventional adaptive quadrature are illustrated for the numerical computation of (1) expected annual loss; and (2) annual rate of collapse. It is shown that for determination of the expected annual loss a 4.5 - to 8.8- fold reduction in the computational demand is obtained using MAQ compared to conventional integration methods. For annual rate of collapse the computational demand reductions range from $30 \%$ to two-fold. The computational reductions are a function of the error tolerance prescribed, with greater computational reductions as stricter tolerances are enforced.
\end{abstract}




\section{KEYWORDS}

Numerical integration; performance-based earthquake engineering (PBEE); magnitudeoriented adaptive quadrature; Seismic loss estimation; Romberg Integration.

\section{INTRODUCTION}

With the rapid growth of performance-based earthquake engineering in research and design, the accurate and efficient evaluation of the governing probabilistic integrals increases in importance. If the functions contained in the integrand are of an appropriate form then it may be possible to obtain a 'closed-form' analytical solution. Such analytical solutions have been presented in the literature, for example: annual rate of exceedance of demand [1-3] and annual rate of structural collapse [4,5]. In general, these 'closed-form' solutions use functions which are local approximations to the true functions defining the arguments of the integral equations. When no simplification is possible without significant loss of accuracy, or the dimensionality of the problem becomes large, these integrals must be solved using a numerical scheme.

Numerical solution of integrals can utilize simulation (e.g. Monte Carlo) or a direct integration technique (e.g. quadrature). Some examples of simulation methods in PBEE include Assembly-Based Vulnerability [6], and Subset Simulation [7]. Direct numerical integration is typically employed to evaluate relationships within the so-called PEER framing equation [8], advocated by the Pacific Earthquake Engineering Research (PEER) Centre. This is because the PEER framing equation makes the Markovian assumption allowing the triple integral to be computed as successive single integrals. It is well realised that unlike quadrature techniques, such simulation methods do not suffer from the 'curse of dimensionality' [9] making their use particularly suited to high-dimensionality problems. As attention herein will be given to solution of equations within the PEER framework formula, 
we will restrict our attention to direct numerical integration methods.

Baker and Cornell [10] propose the use of the first-order second-moment (FOSM) method as a potential method of uncertainty propagation for certain relationships with the PBEE framework. They note however that certain relationships (i.e. those including the ground motion hazard curve) should be used with direct numerical integration (as the ground motion hazard is likely a dominant contributor toward the total uncertainty in the seismic performance measure of interest). Also the FOSM method, being a first-order approximate method, has limitations which may make it significantly inaccurate in certain situations [11].

The use of performance-based frameworks within which these integrals are solved in a practical situation requires that efficient integration algorithms are used offering: (i) numerical efficiency; (ii) accuracy tolerance specifications; and (iii) 'user-friendliness'. Efficiency is of primary importance since the computational demand will influence whether such an approach will be employed. Accuracy tolerance is important as a user should be able to specify a relative error tolerance, and know that the results of the analysis will be accurate to within that tolerance (with the exception of pathological cases). Here we have used the general phrase 'user-friendliness' to indicate the information that is required by the integration algorithm (such as integration region, step size and error tolerances), which is important in the avoidance of pitfalls for inexperienced users, and will be elaborated further on in the manuscript.

Although many standard references are available on numerical solution algorithms, as will be shown in this paper, it is possible to take advantage of the form of the integrand to develop an algorithm with significantly greater efficiency than 'general-purpose' algorithms, and which requires only the integration error tolerance and maximum number of function evaluations (and not any information on step size or integration region). 


\section{THE RISK EQUATIONS}

For brevity, throughout the remainder of this paper the probabilistic integral equations which comprise the PEER framing formula will be referred to as 'the risk equations'.

There are several different appearances of the risk equations, the most well-known being the so-called 'Triple-integral formula' [8] (Eq. (1)), which gives the annual frequency of exceeding some decision variable, $D V$.

$$
\lambda(D V)=\iint \sum G(D V \mid D M)|\Delta G(D M \mid E D P)||d G(E D P \mid I M)| \mid d \lambda(I M
$$

where $I M=$ intensity measure (e.g. PGA); $E D P=$ engineering demand parameter (e.g. peak interstorey drift); $D M=$ damage measure; $D V=$ decision variable; $G(x \mid y)=G(X \geq x \mid Y=y)$ is the complementary cumulative distribution function (CCDF) of $X$ given $Y ; \lambda(z)$ is the annual frequency of exceeding $z ; d G(x \mid y)$ and $d \lambda(z)$ are the differentials of $G(x \mid y)$ and $\lambda(z)$, respectively.

The triple integral formula can be de-coupled into successive single integrals based on the Markovian assumption of one step memory (e.g. in Eq. (1), $G(D V \mid D M)$ is dependent only on $D M$ and not on $E D P$, or $I M)$. Such de-coupling can lead to for example, the rate of exceedance of some level of demand [1-3], and the expected loss given an intensity measure [12], which are given in Eq. (2) and (3), respectively.

$$
\begin{aligned}
& \lambda(E D P)=\int G(E D P \mid I M)|d \lambda(I M)| \\
& E\left[L_{T} \mid I M\right]=\int E\left[L_{T} \mid E D P\right]|d G(E D P \mid I M)|
\end{aligned}
$$

where $E[x \mid y]=$ the expected value of $\mathrm{x}$ given $\mathrm{y}$; and $L_{T}=$ total loss.

Since the Markovian assumption allows a reduction to successive single integrals, then herein we will be concerned with integrals of the following general form: 


$$
I=\int_{\text {all } x} G(x)|d F(x)|=\int_{\text {all } x} G(x)\left|\frac{d F(x)}{d x}\right| d x=\int_{\text {all } x} G(x) f(x) d x
$$

where $G(x)$ and $F(x)$ are piecewise-continuous functions; $d F(x)$ is the differential of $F(x)$; and

$\frac{d F(x)}{d x}=f(x)$ is the derivative of $F(x)$ with respect to $x$. All of the functions comprising the integrand are functions of the integration variable, $x$. As far as the authors are aware, Equation (4) encompasses all of the PBEE equations presented in the literature, with the one known exception that of vector-based equations (e.g. $[13,14])$, in which case $G(x)$ and $F(x)$ are vector-valued functions (i.e. $\boldsymbol{x}=\left[x_{1}, x_{2}, \ldots x_{\mathrm{N}}\right]$ ) and the multiple integration is over the domain of $\boldsymbol{x}$. Such vector-based integrals will not be dealt with explicitly here, although the concepts presented herein are still applicable and are discussed briefly later in the manuscript (as quadrature-based multi-dimensional numerical integration is effectively embedded single numerical integrations). As previously mentioned it should be noted that as the dimensionality of the integrals increases, the efficiency of simulation based procedures will increase relative to that of direct numerical integration (and will eventually become more efficient [9]). As far as the authors are aware, only 2-dimensional vectors have been proposed for the $E D P \mid \mathbf{I M}$ relationship (e.g. $[13,14]$ ), and therefore also 2-dimensional vectors for the $\lambda(\mathbf{I M})$ relationship, but all damage (i.e. $D S \mid E D P$ ) and loss (i.e. $D V \mid D S$ ) relationships are scalar (e.g. $[12,15])$

\section{REGION OF INTEGRATION}

As the integration variable (i.e. $I M$ or $E D P$ ) is defined over all the positive real numbers (i.e. $x \in[0, \infty)$ ), then the integration (strictly speaking) is over this entire domain of the integration variable. However, in practice the magnitude of the integral tends (usually rapidly) to zero at the endpoints of the integration region (a requirement for convergence of the integral as $\mathrm{x}$ tends to infinity, and a practical constraint at $\mathrm{x}$ equals zero), thus allowing a 
sub-region of this domain (instead of the entire domain) to be used to evaluate the integral. For example, in Eq. (3), it is typically assumed that the demand conditioned on intensity, $(E D P \mid I M)$, relationship has a lognormal distribution $[16,17]$. Thus the region of integration can be specified as a certain number of standard deviations either side of the mean demand for the given intensity.

Inadequate selection of the sub-region for integration will lead to underestimation of the value of the integral. It is therefore desired that determination of the integration region be obtained internally within a computational algorithm, thus preventing possible errors by inexperienced users (this is one objective in the previously defined 'user-friendliness'). In certain cases it is not a trivial task to determine the region of integration because of several reasons which are discussed in detail below.

Problems with selection of the integration region can occur when the distribution that is used to 'guess' an appropriate integration region does not conform well to the shape of the integrand. To explain this more clearly we refer to the computation of the mean annual frequency of exceeding some level of demand given in Eq. (2). For this problem the mean and standard deviation of the $E D P \mid I M$, relationship are typically provided via structural analysis instead of $I M \mid E D P$, which requires the use of iteratively scaling ground motion records until a target EDP is achieved [18]. However, for solution of the integral the mean and standard deviation of the intensity given demand $(I M \mid E D P)$ relationship is required in order to determine the appropriate intensity values to integrate over. If it is assumed that locally EDP and IM can be related by $E D P \mid I M=\ln (a)+b \ln (I M)+\beta_{E D P \mid I M} \varepsilon$ (where all terms are deterministic except $\varepsilon$ which has zero expectation and unit lognormal standard deviation)' then the mean and dispersion of the intensity given demand relationship can be given by $\mu_{\ln I M \mid E D P}=\left\lfloor\mu_{\ln E D P \mid M}-\ln (a)\right\rfloor / b$, and $\beta_{\ln I M \mid E D P}=\left(\beta_{\ln E D P \mid M}\right) / b$, respectively [19]. Fig. 1 shows the probability density function (pdf) of the intensity given demand relationship, the 
integrand of the demand hazard for $E D P=0.05$ (where in this case the EDP is assumed to be the peak drift of the structure), and the normalised pdf of the ground motion exceedance (i.e. not the ground motion hazard). Here, seismic intensity and demand are measured using the 1.5 second elastic spectral acceleration $\left(I M=\mathrm{S}_{\mathrm{a}}(\mathrm{T}=1.5 \mathrm{~s}, 5 \%)\right)$, and drift $(E D P=\theta)$, respectively; the $E D P \mid I M$ parameters are $\left(a, b, \beta_{\ln E D P \mid I M}\right)=(0.01,1.5,0.4)$; and the ground motion hazard is for Christchurch, New Zealand [20]. It is immediately evident that the two curves in Figure 1 are offset. If the density of the $I M \mid E D P$ relationship is used to approximate the bounds for the region of integration then for a desired level of accuracy it will likely lead to lower and upper integration limits which are too large. Having a lower limit which is too large will potentially mean that a sub-region of the integration domain which contributes significantly toward the value of the integral will be neglected, resulting in potential significant error. This problem can be resolved by integrating over a large number of standard deviations (i.e. about \pm 4 is sufficient for a integration tolerance of $1 \times 10^{-3}$ ). However in this case, the upper limit will be quite large (using 4 standard deviations gives an upper limit of 8.49) and since the magnitude of the integrand becomes insignificant around an intensity of 5, then integration over this insignificant region (i.e. from intensities of 5-8.5) unnecessarily increases the computational demand.
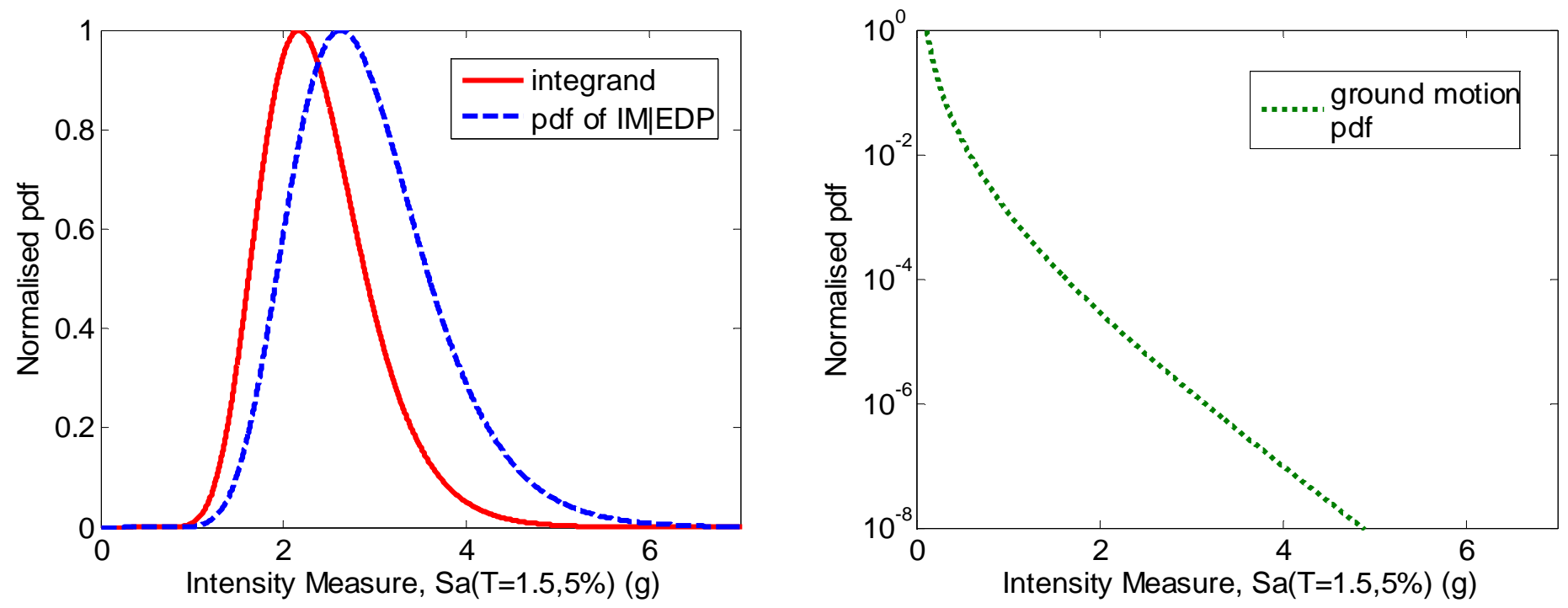
Fig. 1: Illustration of the offset in the probability density function used for integration region estimation with: (a) the integrand of the integral, and (b) the ground motion pdf.

Problems in determining the region of integration can also occur when neither of the two functions comprising the integrand of the risk integral conform to an analytical probability density function (with the exception of extreme value-type distributions). This problem is encountered when trying to compute the expectation of the annual loss (Eq. (3)). In this case the functions in the integrand are the expected loss given intensity and the derivative of the ground motion hazard, neither of which are a 'conventional' probability density. Fig. 2 shows the expected loss given intensity for a typical NZ bridge [21]; ground motion hazard curve for Wellington, New Zealand [20]; and the resulting integrand of the expected annual loss. It can be seen that while it may be relatively simple to determine a subregion of integration from inspection, trying to determine a sub-region within a computational algorithm will require the use of some optimisation algorithm (or similar) to determine where the values of the integrand are or are not significant. This would mean that numerous evaluations of the integrand would be required, none of which would be used toward computing the integral.

In the above two problematic cases it is desirable, instead of predefining a sub-region of integration, to instead integrate over the entire domain of integration (or for the first problem type, using a sub-domain based on a large number of standard deviations), but performing the integration in such a way that little computational effort is expended in computing the integrand over regions which contribute insignificantly toward the numerical value of the integral. An algorithm offering a potential solution to the above statements is addressed in subsequent sections. 

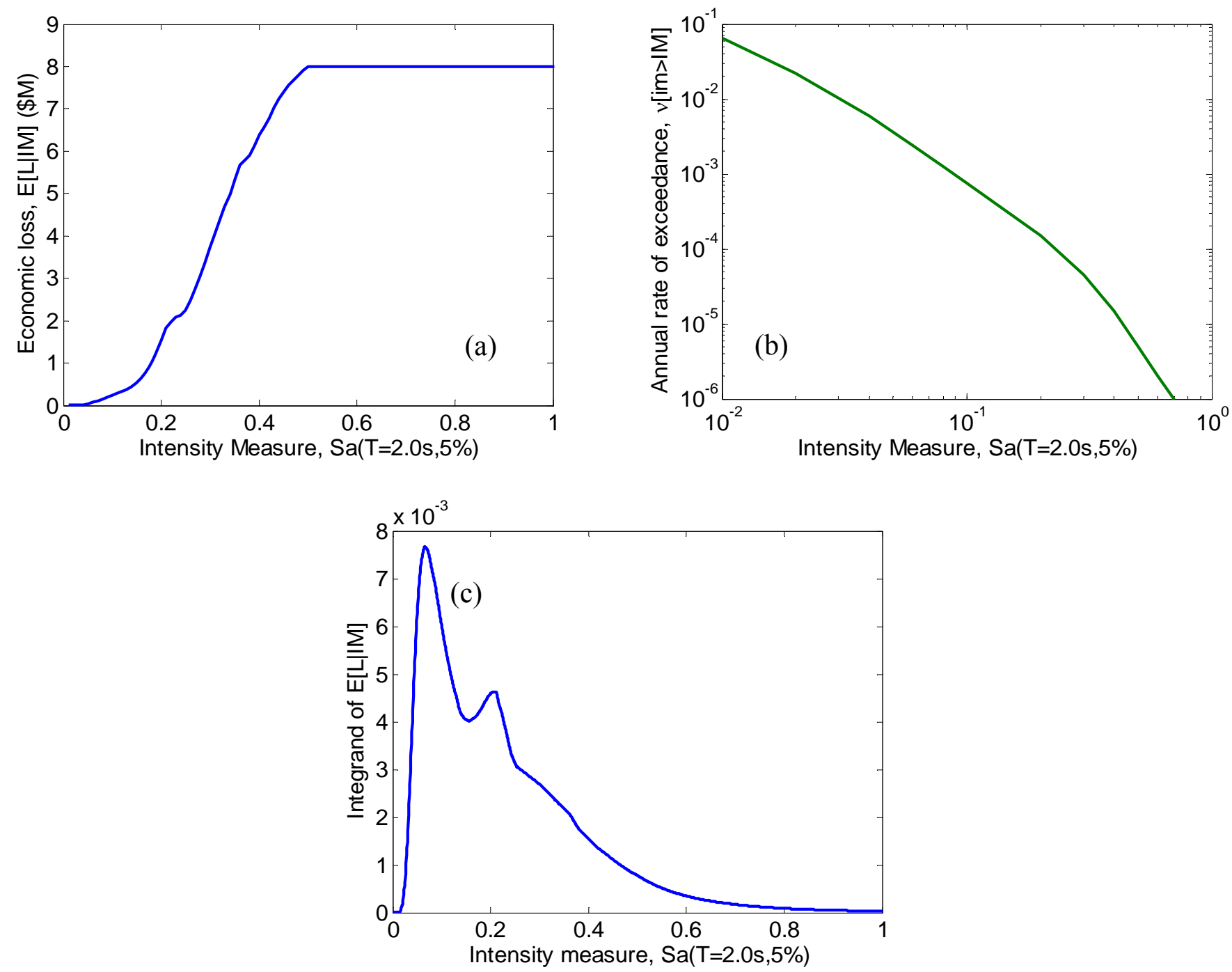

Fig. 2: (a) Expected loss given intensity; (b) ground motion hazard; (c) expected annual loss integrand for the example problem considered.

\section{INDEFINITE LIMITS OF INTEGRATION AND ASSUMPTIONS}

As the domain of $x$ is not finite then Eq. (4) cannot be easily numerically integrated in its current form. There are numerous methods in handling indefinite integrals, in this work the following mapping is used to make the integration domain finite:

$$
t=\frac{1}{(x+1)}
$$

This transformation maps the region $\mathrm{x}=[0, \infty)$ to $\mathrm{t}=(0,1]$, and in particular $\mathrm{x}=0 \rightarrow \mathrm{t}=1$, $x=\infty \rightarrow t=0$. With this transformation, Equation (4) can be re-written the following form: 


$$
I=\int_{x=0}^{x=\infty} G(x) f(x) d x=\int_{t=0}^{t=1} \frac{1}{t^{2}} G(1 / t-1) f(1 / t-1) d t
$$

If the integrand, $G(\mathrm{x}) f(\mathrm{x})$ approachs zero at least as fast as $1 / x^{2}$ as $x$ tends to infinity then the integral converges. This is the same as requiring $G(1 / t-1) f(1 / t-1) \rightarrow 0$ at least as fast as $t^{2} \rightarrow 0$. Eq. (6) is now in a form suitable for direct numerical integration.

We will also make two mild assumptions (which we will later relax) for the forms of the functions comprising the integrand. The first is that the function $G(\mathrm{x})$ is monotonically increasing, and the second that $f(\mathrm{x})$ is a uni-modal function (i.e. it has only one maximum, before which the function is monotonically increasing, and after which it is monotonically decreasing). These two assumptions generally agree with intuition, for example one would expect that as the level of ground shaking (intensity) increases, the level of damage (loss) increases, similarly the function, $f(x)$, is usually a probability density function which is assumed to be lognormal (e.g. the EDP|IM relation) or is similar to an extreme value distribution (e.g. $\lambda(I M))$, both of which are uni-modal functions. Under the above two assumptions it can be shown that while the integrand of $I, G(\mathrm{x}) f(\mathrm{x})$, is not strictly a uni-modal function (although in the majority of cases it will be), the majority of the integral will be contributed from a single sub-region (e.g. Fig. 1 and Fig. 2(c)), and the integrand will not have a significant multi-modal shape (i.e. a significant contribution toward the integral will not come from two or more distinctively separate regions of the integration variable). The premise that the dominant contribution toward the integral will occur over a single region of integration is used to target the computational effort toward the integral evaluation at the location of this region. Nevertheless, the proposed algorithm will cope with significant contributions from several distinct regions provided the initial distribution of integration points is capable of identifying each such region. 


\section{CONCEPT OF MAGNITUDE-ORIENTED ADAPTIVE QUADRATURE}

As previously stated, we aim to have a quadrature method which can adapt around the integrand in order to achieve both accuracy, computational efficiency, and require no integration computation specifics (such as the step size and region of integration), other than the error tolerance and maximum number of function evaluations. For completeness, we first discuss two simple quadrature methods, Romberg integration, and Simpson's rule-based adaptive quadrature, which are globally and locally adaptive methods, respectively. These two methods are described briefly here as they are used as a basis of the locally and globally adaptive quadrature method we propose. Further information on these two algorithms can be found, for example, in $[22,23]$.

One simple but highly efficient algorithm for numerical integration that allows error estimation is Romberg Integration [22]. The Romberg integration algorithm is a computational method of using Richardson Extrapolation with the Trapeziodal rule [23], which uses two approximations (of, in this case, an integral) to compute a third more accurate approximation. The Romberg Integration algorithm can be expressed in the following form:

$$
I_{j, k}=I_{j+1, k-1}+\frac{1}{4^{k-1}-1}\left(I_{j+1, k-1}-I_{j, k-1}\right)
$$

where $k(>1)$ is the order of the approximation error (i.e. $\mathrm{k}=2$ corresponds to $O\left(h^{4}\right), \mathrm{k}=3$ to $O\left(h^{6}\right)$ etc.; $h$ is the step size of integration; the subscript $j$ is used to denoted the more and less accurate approximations of the integral $\left(I_{j+1, k-1}\right.$ and $I_{j, k-1}$, respectively); and $I_{j, k}$ is the improved estimate of the integral. Hence Eq. (7) shows that effectively the improved estimate is obtained by incrementing the more accurate of the two estimates. Romberg integration is efficient in the sense that it is able to use previous integration points when the integration step size is further refined and then combined with Richardson Extrapolation it is markedly more efficient than conventional Simpson's rule [23]. 
The Romberg integration algorithm is most efficient when the curvature of the integrand is relatively constant over the region of integration. When the curvature of the integrand varies significantly over the region of integration, the rate of convergence is reduced because certain sub-regions take longer to achieve convergence (to a specified tolerance) while additional function evaluations are 'wasted' in sub-regions where convergence has already been achieved. The reduction in convergence of the Romberg algorithm in such cases is due to the fact that convergence of the integral is measured globally (i.e. over the entire region of integration).

Adaptive quadratures [22] are one such set of algorithms where convergence is measured locally (i.e. over some sub-region), which makes it more efficient for integrals with rapidly changing curvatures. Adaptive quadrature was initially developed for use in solving ordinary differential equations where so-called 'stiff' problems [22, 23] are encountered. The most common form of adaptive quadrature is that which uses successively refined approximations of Simpson's rule. Simpson's rule-based Adaptive quadrature (herein referred to simply as adaptive quadrature for brevity) is based on the following steps: (1) numerically integrate over some sub-region using Simpson's rule with three points; (2) subdivide the interval by evaluating the integrand at the $1 / 4$ and $3 / 4$ points of the sub-region, then apply Simpson's rule using three points over each of the two half-regions and then add the resulting two values; (3) compare the relative error between the more and less accurate approximations; if the relative error is acceptable then move to the next sub-region, else repeat the process, but using only the left half the original sub-region.

From the previous discussions the following points can be noted for Romberg integration and adaptive quadrature concerning convergence and efficiency. Romberg integration measures convergence globally and therefore efficiency can be reduced when certain regions of the integration domain take longer to converge than others. Adaptive 
quadrature measures convergence locally, therefore overcoming the aforementioned problem of local convergence for Romberg integration. However, because convergence is only measured locally then significant computational effort can be spend evaluating regions of the integration domain whose magnitude does not affect the global convergence. Below an algorithm, herein referred to as magnitude-oriented adaptive quadrature (MAQ), is introduced which aims to provide a solution to both of the noted problems in Romberg integration and adaptive quadrature, which in particular occur in the problem context discussed in this manuscript.

MAQ can be thought of as a further extension of conventional adaptive quadrature. The key differences being that: (i) MAQ considers convergence at both a local and global level; and (ii) MAQ uses a modified region discretization. A schematic illustration of the four-step process of MAQ is given in Fig. 3 and outlined in the following paragraphs. It is also noted that a MATLAB implementation of the MAQ algorithm can be obtained from http://www.civil.canterbury.ac.nz/postgrads/bbradley.asp. Also herein for brevity the region of integration is implied to be the region of integration after transformation by Eq. (5) unless otherwise stated.

Initialisation: To start the algorithm requires the user to specify values for the region of integration (i.e. for $\mathrm{x}=(0$, infinity) $\mathrm{t}=(0,1))$ for the error tolerance, 'tol', and maximum number of function evaluations, 'maxeval'. The initial value of the integral is set to zero as well as counter variables which are used to keep track of the number of function evaluations, and to store computed values in arrays.

Step 1: firstly the integrand is evaluated at the two endpoints ( $a$ and $b$ ) and a midpoint $c=(a+b) / 2$. Based on these three points Simpson's rule is used to estimate the integral over the region of integration $\left(\mathrm{I}_{1}\right)$ which is given by: 


$$
I_{1}=\frac{(b-a)}{6}[f a+4 f c+f b]
$$

where $f a, f b$ and $f c$ denote the value of the integrand at $a, b$ and $c$, respectively.

Step 2: Two additional integrand evaluations are obtained at the points, $d=(c+a) / 2$ and $e=(b+c) / 2$ (which are denoted as $f d$ and $f e$, respectively). Thus, Simpson's rule (i.e. Eq. 8 with the necessary subscript substitutions) can be used to obtain two integral approximations over the regions $(a, c)$ and $(c, b)$. As a smaller step size of integration is used these integral approximations $\left(\mathrm{I}_{2}\right.$ and $\left.\mathrm{I}_{3}\right)$ will be more accurate than the approximation obtained in step 1 $\left(I_{1}\right)$.

Step 3: An approximation of the error between the approximate and exact values of the integral over the sub-region $(\mathrm{a}, \mathrm{b})$ can be obtained as the difference between the more and less accurate approximations $\left(\mathrm{Q}_{1}=\mathrm{I}_{1}\right.$ and $\mathrm{Q}_{2}=\mathrm{I}_{2}+\mathrm{I}_{3}$, respectively). This error is then compared to the local and global convergence criteria to determine if the error is acceptable (local and global convergence criteria are discussed in detail later in the manuscript).

Step 4(i): If convergence is reached for the sub-region considered then the approximation of this particular sub-region is added to the accumulating approximation of the integral. Given that the order of the two approximations $\mathrm{Q}_{1}$ and $\mathrm{Q}_{2}$ are $O\left(h^{4}\right)$ and $O\left([h / 2]^{4}\right)$, respectively, Richardson extrapolation [23] can be used to combine these two estimates to obtain an integral estimate with an error of the order $O\left([h / 2]^{8}\right)$ by:

$$
\Delta I=Q_{2}+\frac{1}{15}\left(Q_{2}-Q_{1}\right)
$$

This high order estimate is the approximation that is added to the accumulating approximation of the integral. As convergence for this sub-region has been reached then the algorithm moves to the next sub-region (i.e. Step 1). 

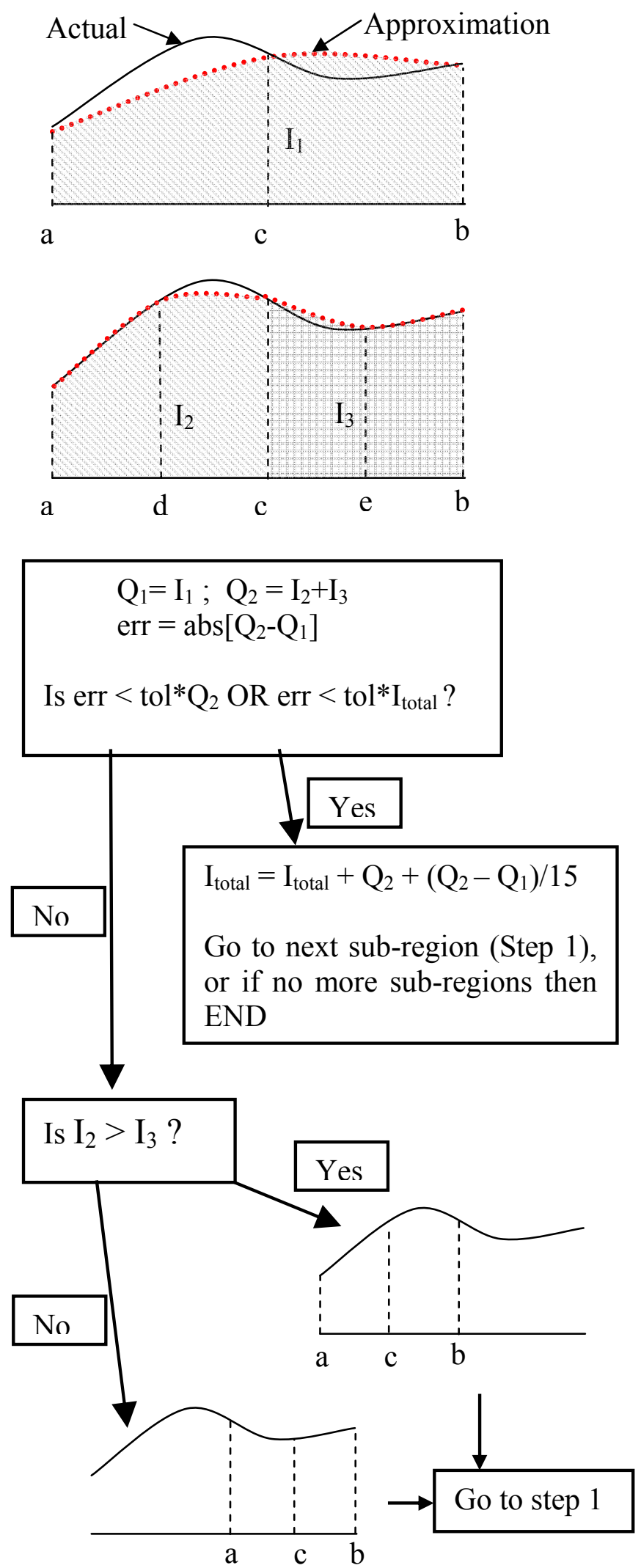

Step 1: Evaluate integrand at points $\mathrm{a}, \mathrm{b}$ and $\mathrm{c}$ and use Simpsons rule to approximate the integral over region $(a, b), I_{1}$

Step 2: Evaluate integrand at points $d$ and e and use Simpsons rule to approximate the integral over region $(\mathrm{a}, \mathrm{c}), \mathrm{I}_{2}$, and over region $(\mathrm{c}, \mathrm{b}), \mathrm{I}_{3}$.

Step 3: Compute error between approximations in steps 1 and 2 and compare error with local and global convergence criteria

Step 4(i): If convergence reached for subregion considered then add increment to accumulating approximation of integral. Then obtain stored data for next sub-region (see step 4(ii)) and return to step 1.

Step 4(ii): If convergence is not reached then select the half of the current sub-region which has the larger integral approximation increment. Store the abscissa and function values for the region which is not selected (these values are retrieved at a later date), and then return to step 1 with the new sub-region.

Fig. 3: Schematic illustration of the four steps in magnitude-oriented adaptive quadrature 
Step 4(ii): If convergence is not reached then the size of the sub-region needs to be reduced (and more function evaluations performed) so that the desired accuracy can be achieved. The sub-region is halved and the choice of which half to select is based on the estimates of the integral over the two half regions. For example, if $I_{2}>I_{3}$ (i.e. the integral over region $(a, c)$ is larger than over $(c, b))$ then region $(a, c)$ is selected as the new sub-region. The abscissa and integrand values computed within the smaller sub-region are stored in an array and the algorithm returns to step 1 with the smaller sub-region. This smaller region is the region which will be the 'next sub-region' described in step 4(i) once convergence is reached in the larger sub-region.

For clarity in Fig. 3, details on the monitoring the number of function evaluations performed relative to the maximum number prescribed, as well as details on storing of data points have been omitted. Further details can be found in the aforementioned programmed version of the algorithm which is available.

From the above four steps it becomes apparent that one of they key aims of the algorithm is to evaluate the integrand primarily around the region which contributes the most toward the integral (this is the effect in step 4(ii) of selecting the larger of the two half regions). This allows the initial computational effort to be focused on efficiently obtaining the dominant contribution to the integral. Fig. 4 illustrates an example problem of the integration of the standard normal density function over the region $\mathrm{x}=(-5,5)$, with an error tolerance of tol $=1 \times 10^{-3}$ using MAQ and adaptive quadrature (note that Romberg integration is not used in the comparison as it is not a locally adaptive method). It is noted for clarity that the standard normal density (SND) has the highest value about $\mathrm{x}=0$ with values tending to zero moving away from this maximum. As conventional adaptive quadrature always selects the left-hand sub-region (see step 4(ii) described previously) when convergence is not reached then adaptive quadrature effectively computes the integral from left-to-right. This is clearly 
illustrated in the accumulating value of the integral, where at the two tails of the SND the contribution to the integral is minor (represented by the initial and final ranges of function evaluations in Fig. 4) and the dominant contribution occurs over a relatively small range of the region of integration (i.e. most of the contribution to the integral comes during a small window of function evaluations). In comparison, the MAQ algorithm targets the evaluation of the integral over this primary region first. As seen in the figure after approximately 20 function evaluations, in which convergence is reached in three sub-regions, the accumulating value of the integral is already $40 \%$ of the final value (indicating most of the converged subregions were around $\mathrm{x}=0$ ).

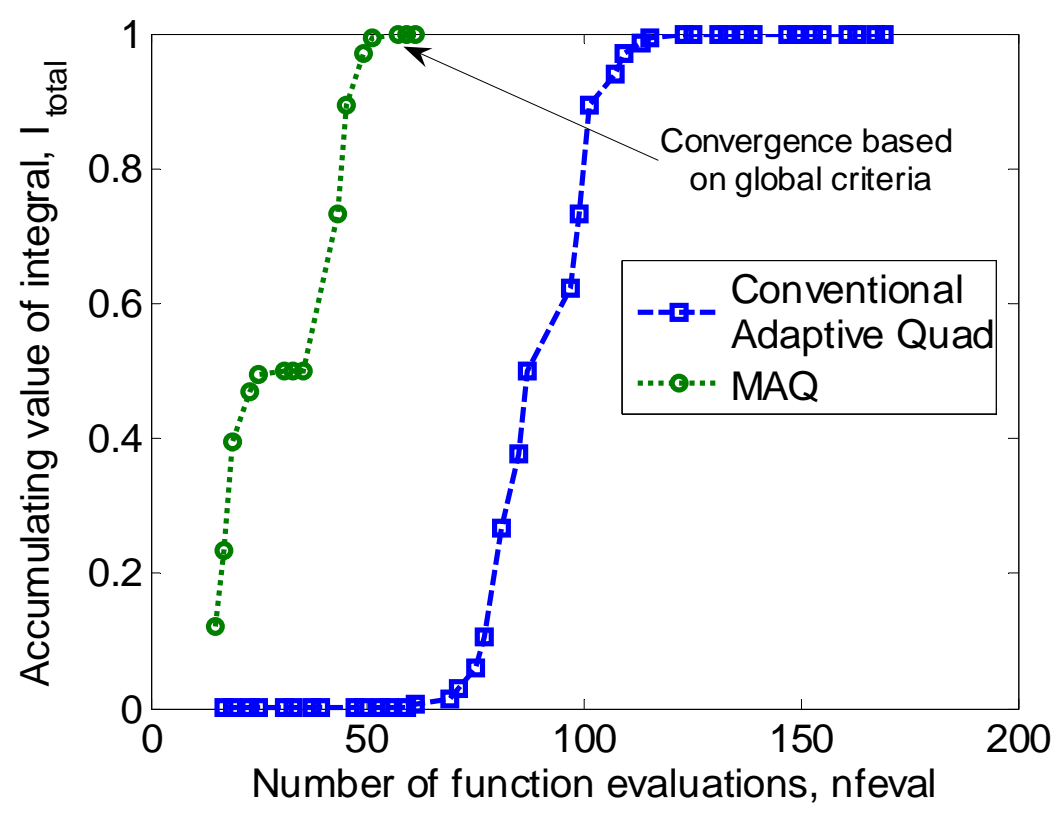

Fig. 4: Example problem illustrating approximation of integral as a function of number of function evaluations.

This targeting of the primary region of contribution to the integral is paramount in allowing the MAQ algorithm to also have global convergence criteria. This global convergence criterion is put in place to allow convergence over regions in which the contribution to the total integral is minor, despite local convergence not being reached. This is achieved by having two separate criteria for convergence (see Step 3 in Fig. 3) only one of 
which is required to be satisfied. The first criterion is that of local convergence based on the truncation error in a single sub-region due to the step size between integration points. The second is that of global convergence which aims to allow convergence of a sub-region in which the integral contribution is minor compared to the final value of the integral. This is shown in Fig. 4 for the MAQ algorithm by the high density of points which do not contribute significantly.

One problem encountered when constructing the MAQ algorithm is that: as the exact value of the integral is not known then how is it possible to determine if integration over some sub-region does or does not contribute significantly to the magnitude of the integral? As the current estimate of the integral is based on the integrand in regions where convergence has been achieved and the integrand is non-negative, this approximation will be less than the true value of the integral (i.e. global convergence of the integral is from below as shown in Fig. 4), then the global tolerance criteria will be conservative. The conservatism is limited in the sense that by the time the less significant regions of the integrand are approached, the majority of the integral has already been computed (and therefore the current accumulating approximation of the integral is likely close to the final value). Thus the only input requirements for the MAQ algorithm are (in general) two error tolerance values (one global and one local) and the maximum number of function evaluations. Herein we will drop the generalisation of using two different error tolerances and use the same error tolerance for both local and global convergence.

\section{EFFICIENCY COMPARISON OF INTEGRATION METHODS}

To illustrate the benefits of the proposed MAQ algorithm over Romberg integration and convential adaptive quadrature, we investigate the two problems that were mentioned in the preceeding sections. 


\section{Case 1: Expected annual loss computation}

Firstly we investigate the case where the region of integration cannot be simply defined as is the case for computation of the expectation of the annual loss. We consider the same problem as referred to in Fig. 2, and consider computation of the integral for relative error tolerances of $10^{-2}$ and $10^{-3}$.

Figure 5 shows six subplots, which illustrate the distribution of the points of function evaluation for the three integration methods using the two different error tolerances. Here the phrase 'nfevals' is used to represent 'number of function evaluations', while 'tol' is used to represent the acceptable error tolerence. It can be seen that using Romberg Integration (Fig. $5(\mathrm{a})$ and $5(\mathrm{~b}))$ a significant number of function evaluations are expended computing the integrand for $\mathrm{t}$ values less than 0.5 (i.e. IM $>1.0$ ). For both tolerance cases, convergence of the integral using Romberg Integration is primarily reduced due to a significant number of function evaluations being required to capture the two peaks of the integrand at $t \sim 0.83$ and 0.94 .

Inspection of the results using adaptive quadrature (Fig. 5a and 5d) illustrates that by measuring convergence locally, over regions of low curvature (such as $t=0.55-0.75$, Fig. 5(c)) the use of adaptive quadrature, as opposed to Romberg Integration can result in significant computational reductions. However, the flaw of measuring convergence only locally results in significant functions evaluations being required to evaluate the integrand for $\mathrm{t}<0.5$, and around the region of $\mathrm{t}=1.0$, despite both of these regions contributing insignificantly toward the integrand. 

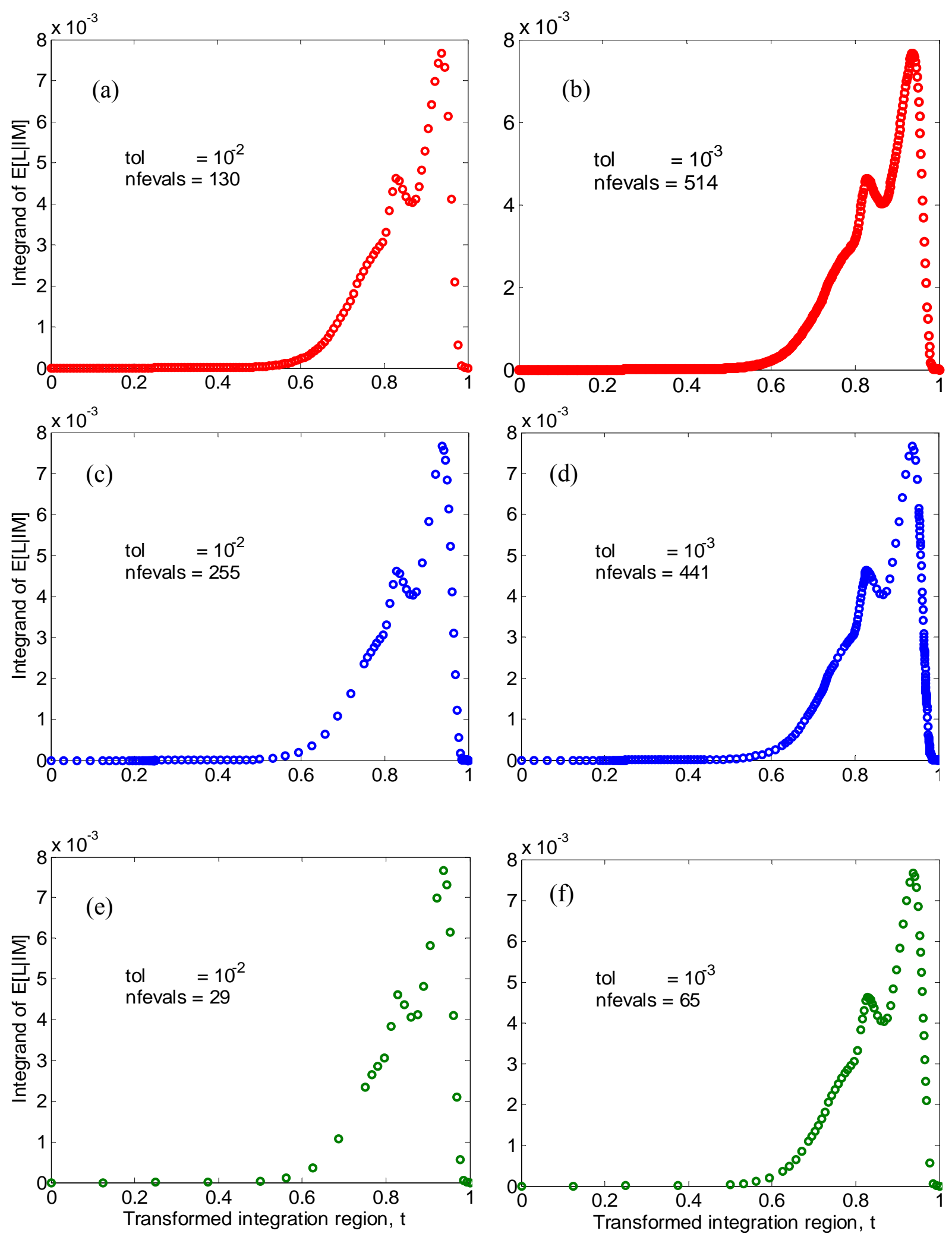

Fig. 5: Illustration of the location of integrand evaluations for computing the expected annual loss problem for (a)\&(b) Romberg Integration; (c)\&(d) Conventional adaptive quadrature; (e)\&(f) MAQ, for error tolerances of $10^{-2}$ and $10^{-3}$. 
Figures 5(e) and 5(f) illustrate the distribution of function evaluations required to compute the integral using MAQ. It can be seen that MAQ does not suffer from the two aforementioned problems exhibited by Romberg Integration and adaptive quadrature. Firstly, over the regions of high curvature $(t=0.77,0.83$, and 0.94$)$ the algorithm adapts locally to reduce the step size around these regions. Secondly, over the insignificant regions of the integrand (i.e. $\mathrm{t}<0.5$ ), MAQ relaxes the local convergence requirements on the basis that the global contribution of this region to the value of the integral is less than the global tolerance of the current value of the integral. The reduction in function evaluations in the insignificant region is particularly evident when the error tolerance is reduced to $10^{-3}$ (Fig. 5(f)). For an error tolerance of $10^{-2}$, the use of MAQ results in a computational reduction of 4.5- and 8.8fold compared to Romberg and Adpative quadrature, respectively. For an error tolerance of $10^{-3}$, the computational reductions are 7.9- and 6.8-fold, respectively.

\section{Case 2: Probability of collapse computation}

To illustrate the computation of a risk integral where one of the integrand arguments is a 'conventional' probability distribution we consider the computation of the annual probability of collapse. As previously mentioned, the fact that one of the arguments of the integrand is a probability distribution allows integration over a specified number of standard deviations, as opposed to integration over the entire domain of the integration variable. Similar to case 1 above, the efficiency (in terms of function evaluations) of the three integration methods is compared for error tolerances of $10^{-2}$ and $10^{-3}$. Therefore the integral is computed based on using endpoints which are three standard deviations either side of the mean (which based on the standard normal distribution will give rise to an error of $2 \times 10^{-3}$, due to neglecting the end regions).

We consider the ground motion hazard shown in Fig. 2(b) and assume that structure has a collapse fragility curve (assumed lognormal) with mean Spectral acceleration 
$(\mathrm{Sa}(\mathrm{T}=2.0 \mathrm{~s}, 5 \%))$ of $0.4 \mathrm{~g}$ and dispersion of 0.3 . As three standard deviations either side of the mean are considered, then the corresponding region of integration is $\mathrm{IM}=\mu \exp \left(-0.5 \beta^{2} \pm 3 \beta\right)=0.085-1.714 \mathrm{~g}$, which corresponds to $\mathrm{t}=0.368-0.922$ (using Eq. (5)).

Figure 6 illustrates the function evaluation distributions for the six different integration permutations. Again, similar to case 1, it can be seen that Romberg integration (Fig. 6(a) and 6(b)) requires additional functional evaluations about the peak of the integrand to achieve global convergence, and as a result a significant number of function evaluations are expended over the tails of the integrand. Integration using adaptive quadrature (Fig. 6(c) and 6(d)) captures the primary region of the integrand efficiently, but the local convergence criteria means that significant function evaluations are required over the tails of the distribution. Again, the use of MAQ for this problem allows efficient integration over the entire integration domain. For a tolerance of $10^{-2}$, MAQ requires approximately only two-thirds of the function evaluations that are required using the other two methods. The efficiency of MAQ is particularly evident when the error tolerance is decreased to $10^{-3}$ (Fig. 6(e) and 6(f)). Using MAQ, only $8(\sim 40 \%)$ additional function evaluations are required to achieve convergence for an error tolerance of $10^{-2}$, while the number of function evaluations almost doubles for both Romberg Integration and Adaptive Quadrature. 

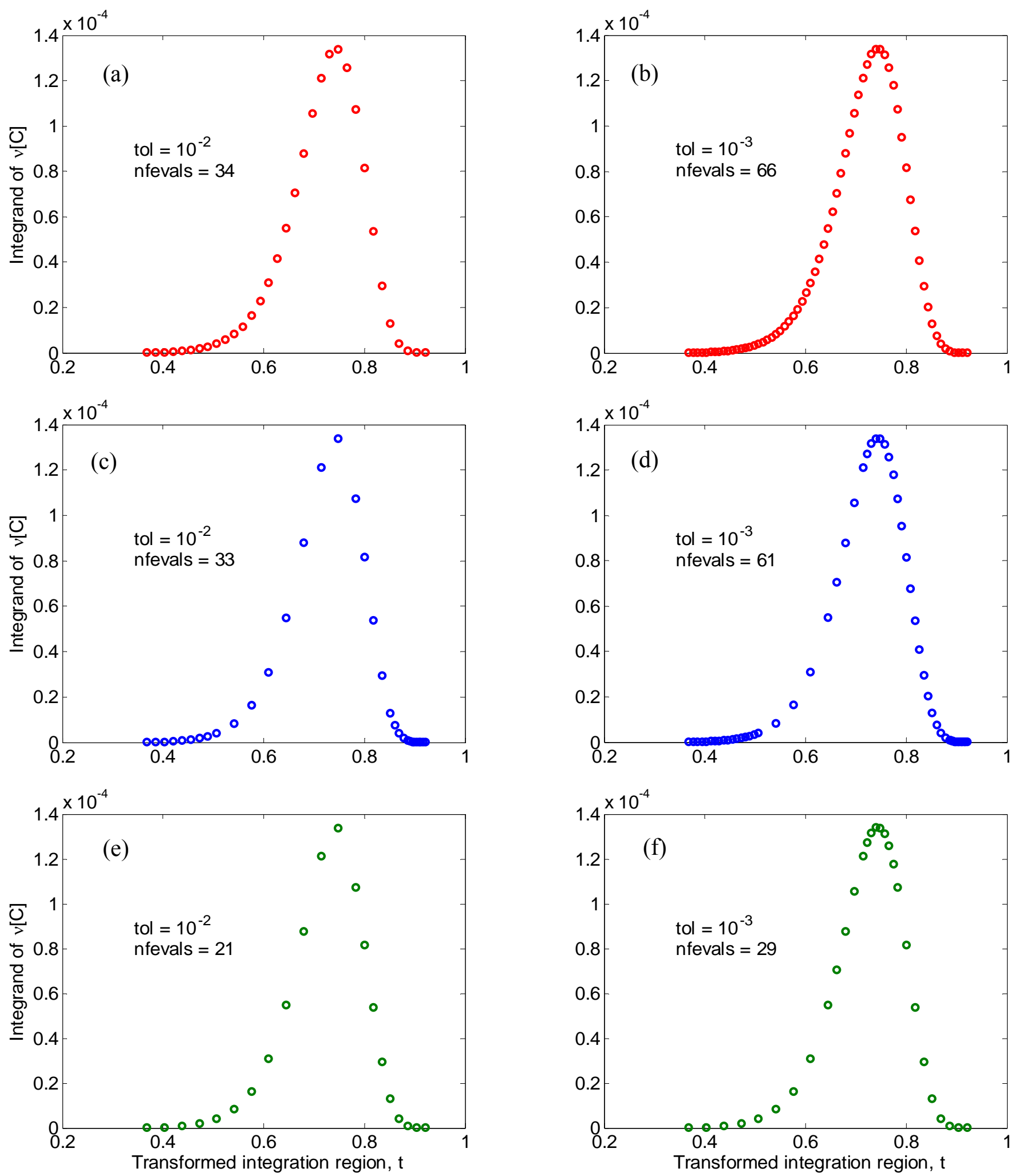

Fig. 6: Illustration of the location of integrand evaluations for computing the annual rate of collapse problem using 5 standard deviations for (a)\&(b) Conventional adaptive quadrature; (c)\&(d) Romberg Integration; (e)\&(f) MAQ, for error tolerances of $10^{-2}$ and $10^{-3}$. 
It should also be noted although not shown here, that if a higher accuracy solution is desired then the number of standard deviations to integrate over must be increased. This would result in substantially more computational demand for the Romberg and Adaptive quadrature algorithms, while the increase in computational demand for the MAQ algorithm would be only minor. The reason that the increase would be minor comes back to the idea that MAQ is efficient at handling these tails of the integrand (such as that shown Fig. 4(f) for $\mathrm{t}<0.5)$

The MAQ algorithm can also be extended to the numerical evaluation of multiple integrals, in which it would be significantly more efficient compared the other two alternatives discussed in this papers since the computational work for multiple integrals can be

approximated as $\overline{\text { nfeval }}^{N}$, where $\overline{\text { nfeval }}$ is the average number of function evaluations in each dimension, and $\mathrm{N}$ is the order/dimension of integration (i.e. single $=1$, double $=2$ ). Thus a two-fold reduction in computational work for a single integral is equivalent to a 4-fold reduction for a similar double integral.

\section{CONCLUSIONS}

An integration algorithm called Magnitude-oriented Adaptive Quadrature (MAQ) has been developed specifically for integration of the governing equations within the PEER framework formula. It has been illustrated that in addition to the methods being significantly more efficient than conventional integration algorithms such as Romberg integration and Simpson's-rule based Adaptive Quadrature, MAQ is also 'user-friendly' in the sense that only the error tolerance is required to be specified (and not any information on the region of integration etc.). Therefore it can be conclusively stated that a seismic loss estimation framework which propagates uncertainty through direct numerical integration with MAQ will be significantly more efficient than one which uses conventional integration algorithms. 
Although attention on the application of the proposed MAQ algorithm in this manuscript has been given only to the PEER framing equations, there is no reason why the algorithm cannot be used in other situations. It will likely be an efficient integration algorithm for problems which contain integrals with a non-negative integrand of a similar functional form to those discussed here.

Based on the findings of this research the following conclusions can be made:

1. The determination of an adequate yet efficient sub-region of integration for PBEE integrals over the entire domain of positive real numbers is a challenging task.

2. An algorithm is presented which allows integration over the entire integration domain, with computational effort focused on those regions which provide the majority of the contribution of the magnitude of the integral

3. Through two case examples it was shown that the computational reduction is reduced can range from a 4.5- to 8.8-fold in the case of integration over the entire domain, and a $30-100 \%$ reduction when only part of the integrand is required to be integrated, compared to Romberg and Adaptive quadrature algorithms.

\section{ACKNOWLEDGEMENTS}

Financial support of the first author by the New Zealand Tertiary Education Commission is greatly appreciated.

\section{REFERENCES}

[1] McGuire RK. Seismic Hazard and Risk Analysis. Earthquake Engineering Research Institute: EERI MNO-10; 2004.

[2] Cornell CA. Reliability-Based Earthquake-Resistant Design - the Future. In: Proceedings $11^{\text {th }}$ World Conference on Earthquake Engineering; 1996. 
[3] Kennedy RP, Short SA. Basis for Seismic Provisions of Doe-Std-1020. Ucrl-Cr-111478 and Bnl-52418. Lawrence Livermore National Laboratory and Brookhaven National Laboratory; 1994.

[4] Shome N, and Cornell CA. Probabilistic seismic demand analysis of nonlinear structures. Report No. RMS-35, RMS Program, Stanford University, Stanford, CA 1999.

[5] Zareian F, Krawinkler H. Assessment of probability and design for collapse safety. Earthquake Engineering and Structural Dynamics 2007; (in press).

[6] Porter KA, Kiremidjian AS, LeGrue JS. Assembly-Based Vulnerability for Buildings and Its Use in Performance Evaluation, Earthquake Spectra 2001;17(2), pp. 291-312.

[7] Au SK, Beck JL. Subset Simulation and its Application to Seismic Risk based on Dynamic Analysis. Journal of Engineering Mechanics 2003; 129(8), pp. 901-917.

[8] Deierlein GG, Krawinkler H, Cornell CA. A Framework for Performance-based Earthquake Engineering. Pacific Conference on Earthquake Engineering. 2003. Christchurch, New Zealand.

[9] Evans M, Swartz T. Methods for Approximating Integrals in Statistics with Special Emphasis on Bayesian Integration Problems. Statistical Science 1995; 10(3), pp. 254-272.

[10] Baker JW, Cornell CA. Uncertainty Specification and Propagation for Loss Estimation using FOSM Method. Peer Report No 2003/07. September 2003. http://peer.berkeley.edu/publications/peer reports.html

[11] Bradley BA, Lee DS. Accuracy of Approximate Methods of Uncertainty Propagation in Loss Estimation. Structural Safety (in review).

[12] Aslani H. Probabilistic Earthquake Loss Estimation and Loss Disaggregation in Buildings. Ph.D. Thesis 2005, John A. Blume Earthquake Engineering Centre, Dept. of Civil and Environmental Engineering, Stanford University, Stanford, CA. 382 pages

[13] Baker J, Cornell CA. A vector-valued ground motion intensity measure consisting of spectral acceleration and eplison. Earthquake Engineering and Structural Dynamics 2005;34(10):1193-1217

[14] Bazzurro P. Probabilistic seismic demand analysis, Stanford University, Stanford, CA, 1998;329 pp. http://www.stanford.edu/group/rms/ 
[15] Mitrani-Reiser J. An Ounce of Prevention: Probabilistic Loss Estimation for Performance-based Earthquake Engineering. PhD Thesis 2007. California Institute of technology, CA 173pp.

[16] Mander JB, Dhakal RP, Mashiko N, Solberg KM. Incremental dynamic analysis applied to seismic financial risk assessment of bridges. Engineering Structures. 2007;29(10):2662-2672.

[17] Aslani H, Miranda E. Probability-based Seismic Response Analysis. Engineering Structures 2005;27(8):1151-1163.

[18] Luco N, Cornell CA, Yeo GL. Annual limit state frequencies for partially-inspected earthquake-damage buildings. Structural Safety 2002;24(2-4):281-296

[19] Benjamin J. and Cornell C.A., Probability, statistics, and decision for civil Engineers. New York: McGraw-Hill; 1970

[20] Bradley BA, Dhakal RP, Cubrinovski M, Mander JB, MacRae GA. Improved Seismic Hazard Model with Application to Probabilistic Seismic Demand Analysis, Earthquake Engineering and Structural Dynamics 2007;36(14):2211-2225.

[21] Bradley BA, Dhakal RP, Mander JB. Parametric Structure Specific Seismic Loss Estimation", $4^{\text {th }}$ International Conference on Urban Earthquake Engineering, Tokyo, Japan. Keynote Lecture. 2007; pp45-52.

[22] Kythe PK, and Schaferhotter MR. Handbook of Computational Methods for Integration. Chapman and Hall/CRC 2000.

[23] Chapra SC, and Canale RP. Numerical Methods for Engineers: with software and programming applications. Fourth Edition. McGraw-Hill 2002. 